\title{
Equivalence between S-Systems and Volterra Systems
}

\author{
EBERHARD O. VOIT AND MICHAEL A. SAVAGEAU \\ Department of Microbiologv and Immunologv, The University of Michigan, Ann Arbor. \\ Michigan 48109
}

Re'cived 28 June 1985; revised 2 August 1985

\begin{abstract}
$S$-systems and Volterra systems have been developed independently of each other in different branches of biology. We show that these systems are mathematically equivalent.
\end{abstract}

\section{INTRODUCTION}

Two decades ago, the need for a nonlinear description of biological and other organizationally complex synergistic systems initiated the development of the power-law formalism [10-16]. In this formalism, the change of a real system in time is assumed to be governed by logarithmically differentiable functions. These are approximated by the constant and linear terms of their Taylor series in logarithmic space, which yields, independently of the specific mathematical features of the approximated functions, products of power functions in Cartesian space. Mathematically as well as biologically most fruitful and accurate is a variant of the power-law formalism in which the functional description of all processes that contribute to the synthesis of a system component and all those that contribute to its degradation are first "aggregated" into one functional description each and then approximated. The result is a system of nonlinear differential equations that has been called an $S$-system:

$$
\dot{X}_{i}=\alpha_{i} \prod_{j=1}^{n} X_{j}^{g_{i j}}-\beta_{i} \prod_{j=1}^{n} X_{j}^{h_{i j}}, \quad i \in\{1, \ldots, n\}
$$

The variables $X_{i}$ are defined as positive real quantities, the parameters $\alpha_{i}$ and $\beta_{i}$ are positive real, and the double-indexed parameters $g$ and $h$ can be any real numbers. An enormous variety of phenomena from many different 
areas of biology have been described and analyzed as $S$-systems (e.g. [16, 22, 3]).

$S$-system descriptions of biological phenomena have an amazingly wide range of validity over variation in the system's constituents. Several types of explanations and justifications for the validity and accuracy of the formalism have been proposed elsewhere $[16,21,25]$. One argument is that a host of differential equations and "laws" in the natural sciences can be recast equivalently as $S$-systems. Prominent examples are linear systems [26], probability functions [20], and growth laws [17, 19], which have been shown to be special cases of a two-variable $S$-system, many physical laws, for instance those describing electrical circuits, gravitation, and cooling and dilution processes [24], and to the so-called special functions in classical physics like those by Bessel, Chebyshev, and Laguerre [24]. In fact, we have not yet encountered an ordinary differential equation that we could not recast as an $S$-system. This variety of laws that are mathematically equivalent to $S$-systems demonstrates that $S$-systems capture essential features of physical phenomena and suggests considering the $S$-system as a general formalism that underlies many processes in nature.

Another approach to modeling biological systems uses Volterra systems [6]:

$$
\dot{N}_{i}=N_{i}\left(a_{i}-\sum_{j=1}^{m} p_{i j} N_{j}\right), \quad i \in\{1, \ldots, m\}
$$

The variables $N_{i}$ are defined as positive real, whereas the parameters $a_{i}$ and $p_{i j}$ can be any real numbers. Originally designed to describe populations in terms of interactions between a predator and a prey [5, 27], Volterra systems now are used for modeling a variety of phenomena in ecology [6] and epidemiology [1, 7] and also in physics (cf. [2]). Mathematical treatments can be found in [6] and $[8,9]$ and the literature cited therein.

A comparison between Equations (1) and (2) shows that Volterra systems emphasize the additive nature of biological systems, whereas $S$-systems tend to emphasize their multiplicative nature. Therefore, these systems appear to be rather different, except for the original special case with one predator and one prey species [17]. In this report, we show that $S$-systems and Volterra systems are in fact mathematically equivalent. By "equivalent" we shall mean that any Volterra system can be transformed into an $S$-system that has exactly the same solution and that any $S$-system can be transformed into a Volterra system with exactly the same solution. These "equivalent" representations can be considered as descriptions of the same process in two different languages, the Volterra-system formalism and the $S$-system formalism. 


\section{RESULT}

\section{THEOREM}

S-systems and Volterra systems are equivalent.

Proof.

(a) Each $S$-system can be formulated as a Volterra system. Starting with the original $S$-system (1), we define the variables $Z_{1}, \ldots, Z_{2 n}$ as

$$
Z_{i}=\mu_{i} \prod_{k=1}^{n} X_{k}^{f_{i k}}
$$

with

$$
\mu_{i}= \begin{cases}\alpha_{i}, & i \in\{1, \ldots, n\} \\ -\beta_{i-n}, & i \in\{n+1, \ldots, 2 n\}\end{cases}
$$

and

$$
f_{i k}= \begin{cases}g_{i k}-\delta_{i k}, & i \in\{1, \ldots, n\}, \\ h_{i-n, k}-\delta_{i-n, k}, & i \in\{n+1, \ldots, 2 n\},\end{cases}
$$

where $\delta_{i k}$ is the Kronecker symbol

$$
\delta_{i k}= \begin{cases}0, & i \neq k \\ 1, & i=k\end{cases}
$$

Differentiation of $Z_{i}$ in Equation (3) yields

$$
\begin{aligned}
\dot{Z}_{i} & =\mu_{i} \sum_{j=1}^{n} f_{i j} \dot{X}_{j} X_{j}^{-1} \prod_{k=1}^{n} X_{k}^{f_{i k}} \\
& =Z_{i} \sum_{j=1}^{n} f_{i j} \dot{X}_{j} X_{j}^{-1} \\
& =Z_{i} \sum_{j=1}^{n} f_{i j}\left(\alpha_{j} X_{j}^{-1} \prod_{k=1}^{n} X_{k}^{g_{j k}}-\beta_{j} X_{j}^{-1} \prod_{k=1}^{n} X_{k}^{h^{\prime k}}\right) \\
& =Z_{i} \sum_{j=1}^{n} f_{i j}\left(Z_{j}+Z_{n+j}\right) .
\end{aligned}
$$

If we define for $i, j \in\{1, \ldots, 2 n\}$

$$
e_{i j}= \begin{cases}f_{i j}, & j \in\{1, \ldots, n\}, \\ f_{i, j-n}, & j \in\{n+1, \ldots, 2 n\},\end{cases}
$$


then we obtain

$$
\dot{Z}_{i}=Z_{i} \sum_{j=1}^{2 n} e_{i j} Z_{j}, \quad i \in\{1, \ldots, 2 n\} .
$$

Equation (5) is a Volterra system with $m=2 n$ and $a_{i}=0$ [cf. Equation (2)]. The number of equations has increased from $n$ to $2 n$. The number of parameters $e_{i j}$ formally is $4 n^{2}$. However, since only half of these parameters are unique, in number they are the same as the number of $g$ plus $h$ parameters in Equation (1). The parameters $\alpha_{i}$ and $\beta_{i}$ do not appear in Equation (5) explicitly, but specify the initial conditions $Z_{i 0}$ [cf. Equation (3)]:

$$
Z_{i 0}= \begin{cases}\alpha_{i} X_{i 0}^{-1} \prod_{k=1}^{n} X_{k, k}^{g, k}, & i \in\{1, \ldots, n\}, \\ -\beta_{i-n} X_{i-n, 0}^{-1} \prod_{k=1}^{n} X_{k 0}^{h i j, k}, & i \in\{n+1, \ldots, 2 n\} .\end{cases}
$$

Although the number of parameters plus initial conditions appears to be $2 n^{2}+3 n$ in Equation (1), but only $2 n^{2}+2 n$ in Equation (5), it is known that there are $n$ redundant parameters in Equation (1). With suitable scaling, the new parameters $\alpha_{i}$ equal $\beta_{i}[16,24]$, and thus the numbers of independent parameters plus initial conditions are exactly the same in Equations (1) and (5).

In fact, both representations describe manifolds of the same dimension. This can be seen most easily from the nonzero steady-state solutions of fully determined systems. In the $S$-system formalism, they are obtained by inverting the $S$-system equations, which are linear in logarithmic space; a unique solution is obtained because the rank of the $n \times n$ matrix $\left(g_{i j} \quad h_{i j}\right)$ is cqual to $n$ for a determined system [16]. The steady-state solution of the corresponding Volterra system is obtained from $2 n$ equations. However, the $2 n \times 2 n$ system matrix is underdetermined and has the same rank as the $S$-system matrix, $n$. The constraints provided by the transformation and the initial conditions ensure that the solution to the Volterra system is uniquely determined and identical to that of the original $S$-system.

(b) Each Volterra system can be formulated as an S-system. It has to be shown that the $i$ th equation of the Volterra system can be written as a difference of products of power functions. This can most directly be seen if we define the trial solution

$$
N_{i}=\exp \left(a_{i} t\right) \prod_{k=1}^{m} W_{i k}^{-1}
$$


with variables $W_{i k}$ that have to be specified. A motivation for this kind of definition will be presented elsewhere [26]. The double subscripts are introduced for convenience. Differentiating and substituting Equation (6) in Equation (2) yields

$$
\begin{aligned}
a_{i} \exp \left(a_{i} t\right) \prod_{k=1}^{m} W_{i k}^{-1}+\exp \left(a_{i} t\right) \sum_{j=1}^{m}\left(-\dot{W}_{i j} W_{i j}^{-1}\right) \prod_{k=1}^{m} W_{i k}^{-1} \\
\quad=\exp \left(a_{i} t\right) \prod_{k=1}^{m} W_{i k}^{-1}\left(a_{i}-\sum_{j=1}^{m} p_{i j} \exp \left(a_{j} t\right) \prod_{k=1}^{m} W_{j k}^{-1}\right),
\end{aligned}
$$

which reduces to

$$
\sum_{j=1}^{m}\left(\dot{W}_{i j} W_{i j}^{-1}-p_{i j} \exp \left(a_{j} t\right) \prod_{k=1}^{m} W_{j k}^{-1}\right)=0
$$

A solution of the following set of differential equations (9) is a solution to Equation (8):

$$
\begin{aligned}
& \dot{W}_{j 0}=a_{j} W_{j 0} \\
& \dot{W}_{i j}=p_{i j} W_{j 0} W_{i j} \prod_{k=1}^{m} W_{j k}^{-1}, \quad i, j \in\{1, \ldots, m\} .
\end{aligned}
$$

Equation (9) is a special type of $S$-system in which each equation contains only one product of power functions and either $\alpha$ or $\beta$ is equal to zero [cf. Equation (1)]. The variables in the $n=m^{2}+m$ equations can be identified as

$$
\begin{aligned}
X_{j} & =W_{j 0}, \\
X_{m i+j} & =W_{i j}, \quad i, j \in\{1, \ldots, m\} .
\end{aligned}
$$

The parameters $a_{i}$ and $p_{i j}$ in Equation (9) correspond to either an $\alpha_{i}$ or a $\beta_{i}$ parameter, depending on whether the $a_{i}$ and $p_{i j}$ are positive or negative; all $g$ and $h$ parameters are either 1,0 , or -1 .

The initial conditions in Equation (9) are highly redundant. $m^{2}$ among them can be chosen arbitrarily, and then the remaining $m$ are uniquely determined. For instance, at the initial time $t_{0}$ we can specify that

$$
W_{i j}\left(t_{0}\right)=1, \quad j \in\{0, \ldots, m-1\},
$$

and then the remaining initial conditions are given uniquely by

$$
W_{i m}\left(t_{0}\right)= \begin{cases}\exp \left(a_{i} t_{0}\right)\left[N_{i}\left(t_{0}\right)\right]^{-1}, & N_{i}\left(t_{0}\right) \neq 0 \\ 0, & N_{i}\left(t_{0}\right)=0\end{cases}
$$


With such a definition, the number of independent parameters plus initial conditions, $m^{2}+m$, is again the same for the original Volterra system (2) and the transformed system (9).

Two features of the transformations between Volterra systems and $S$-systems should be mentioned:

(1) Equation (6) for $W_{i j}$ is almost "inverse" to Equation (3), particularly if all parameter values $a_{i}$ are equal to zero.

(2) Transformation of a general $S$-system yields a special Volterra system $\left(a_{i}=0\right)$, and transformation of a general Volterra system yields a special $S$-system $\left(\alpha_{i}\right.$ or $\left.\beta_{i}=0\right)$. If a general Volterra system is transformed into an $S$-system and this $S$-system is transformed into a Volterra system, all constants $a_{i}$ of the resulting Volterra system are equal to zero. This shows that the general Volterra system is mathematically equivalent to a Volterra system with $a_{i}=0$ (cf. [8]). Similarly, transformation of a general $S$-system into a Volterra system and transformation of this Volterra system into an $S$-system eliminates the $\alpha$-term or $\beta$-term in each equation of the $S$-system, showing that the general $S$-system is equivalent to an $S$-system with either $\alpha_{t}$ or $\boldsymbol{\beta}_{i}=0$ (cf. [26]).

\section{DISCUSSION}

It has long been realized that models for interactions between species in ecology and for chemical reactions show a close similarity on the structural level. Both can be formulated in terms of populations (of organisms or molecules), interactions or collisions (between predator and prey or between molecules), and intermediate complexes (hosts infected with parasites or enzyme-substrate complexes), and both can be represented by the same type of schematic diagram with pools and arrows. Nevertheless, the $S$-system and the Volterra system, which have developed to model these structurally similar phenomena in biochemistry and ecology, respectively, have appeared to be quite distinct, except for the original one-predator one-prey system $[17,19]$ and other trivial special cases. Because Volterra systems are formulated as sums and $S$-systems as products, they have seemed to represent different parts of the nonlinear world. We have now shown that $S$-systems and Volterra systems are mathematically equivalent. Does that mean that one or the other of these formalisms henceforth will be obsolete? Several arguments oppose this conclusion.

These formalisms have been developed in different areas of biology: Volterra systems in ecology, $S$-systems in biochemistry, genetics, and cellular and molecular biology. Both are established entities within these fields, and their variables and parameters have assumed particular meanings such as numbers of individuals, proliferation rates, interaction terms in Volterra 
systems of ecology, and concentrations, apparent kinetic orders, and rate constants in $S$-systems describing biochemical networks. If one system is transformed into the other, variables and parameters are redefined, aggregated, or separated. As a consequence, variables and parameters in the "new" systems have a different meaning and cannot be directly interpreted as before. Because language and terminology influence thinking, this loss of the old parameters might be considered a negative consequence. However, such rearrangement might have positive consequences. It might yield new insight and, in fact, lead to parameters that can cxpcrimentally be measurcd or have a meaning that is easily understood, whereas the parameters of the "old" system were aggregate measures including many parts of the system and hence were difficult to obtain experimentally.

"Recipes" have been developed for translating a biological phenomenon into an $S$-system [16, 23]. Similarly, one can translate proliferation and death rates and interactions of populations into the variables and parameters of a Volterra system. However, since Volterra systems emphasize the additive nature of a phenomenon but $S$-systems aggregate interactions into products, the outcomes will generally be different. Again, this duality might yield fruitful insight and can lead to interesting results if a Volterra system is not compared with the mathematically equivalent $S$-system but with the $S$-system that results from applying the translation recipe. In particular, the duality raises the question of accuracy of different approximations and consequently the question of optimal strategies for modeling biological phenomena.

In some situations, one or the other formalism may be easier to understand or may present a more intuitive way for including well-established "laws." For example, allometric relationships, which constitute an almost universal law underlying organismic growth, are readily seen in the $S$-system formalism [18], whereas they are not obvious in the formalism of the Volterra system.

Mathematical equivalence in the sense we have used this term does not necessarily mean that the systems are equal in every aspect. One important difference is their mathematical and computational tractability. Both systems have a very clear structure that implies specific numerical techniques that take advantage of these structures. An efficient program to solve and analyze $S$-systems already has been developed [4] and it has been shown for a variety of systems that more efficient solutions can be obtained by transformation into $S$-systems. A similar program has been developed for solving Volterra systems and is expected to be particularly efficient for systems that are primarily additive rather than multiplicative.

These arguments show that it is useful to maintain both formalisms as separate entities. Nevertheless, the equivalence between these systems is of great importance. It allows us to study one system with methods of analysis 
developed for the other. The equivalence also unites the ranges of application and classes of special cases of each formalism, thus increasing the power and generality of both. Now it should be possible to translate the mathematical properties of one system and all the theorems related to it into the other formalism. Mathematical analysis of a particular problem then can utilize results and methods from each formalism. For certain steps, one representation might be used because of its advantages, and then one would transform the problem into the alternative formalism whenever it is advantageous to do so.

All equations that can be recast in one formalism can now be recast in the other. Once equations can be recast in the same formalism, a rational system of classification and taxonomy becomes possible. Such a classification is a first step toward a general understanding of nonlinear differential equations and might yield criteria for solvability in terms of elementary functions.

Furthermore, since very many, if not all, ordinary differential equations can be recast as $S$-systems, the formalism of $S$-systems and Volterra systems appears to provide the basis for a very general nonlinear systems theory. As of yet, the most powerful theory of differential equations is strictly linear. Both, Volterra systems and $S$-systems, are nonlinear but have some features that are very similar to linear systems: In Volterra systems, the nonzero steady state is obtained by the solution of a linear algebraic system; in $S$-systems, the nonzero steady state also is obtained by the solution of a linear algebraic system, which is produced by a simple logarithmic transformation [16]. The $S$-system formalism contains linear systems as a special case [26] and hence is an extension of the linear theory into the nonlinear domain. Thus, the formalism of $S$-systems and Volterra systems is a structure that is not unrelated to the existing linear theory, but the linear theory is naturally embedded in the emerging nonlinear systems theory based upon $S$-systems and Volterra systems.

This work was supported in part by U.S. Public Health Service grant GM 30054 to M. A. S. from the National Institutes of Health. We thank D. Irvine, $B$. Palsson, and C. Simon for criticism of the original manuscript.

\section{REFERENCES}

1 R. M. Anderson and R. M. May, Population biology of infectious diseases. Nature 280:361-367 (1979).

2 A. Arneodo, P. Coullet, and C. Tresser, Occurrence of strange attractors in threedimensional Volterra equations, Phys. Lett. 79A:259-263 (1980).

3 D. H. Irvine and M. A. Savageau, Network regulation of the immune response: Alternative control points for suppressor modulation of effector lymphocytes, $J$. Immunol. 134:2100-2116 (1985).

4 D. H. Irvine and M. A. Savageau, in preparation. 
5 A. J. Lotka, Elements of Physical Biology, Williams and Wilkins, Baltimore, 1924: reprinted as Elements of Mathematical Biology, Dover, New York, 1956.

6 R. M. May, Stability and Complexity in Model Ecosvstems, Princeton U.P., Princeton, N.J., 1973.

7 R. M. May and R. M. Anderson, Epidemiology and genetics in the coevolution of parasites and hosts, Proc. Roy. Soc. London Ser. B, 219:281-313 (1983).

8 K. Redheffer and $Z$. Zhiming, Global asymptotic stability for a class of many-variable Volterra prey-predator systems, Nonlinear Anal. Theor. Meth. Appl. 5(12):1309-1329 (1981).

9 R. Redheffer and W. Walter, Solution to the stability problem for a class of generalized Volterra prey-predator systems, J. Differential Equations 52(2):245-263 (1984).

10 M. A. Savageau, Biochemical systems analysis I. Some mathematical properties of the rate law for the component enzymatic reactions, $J$. Theoret. Biol. 25:365-369 (1969).

11 M. A. Savageau, Biochemical systems analysis II. The steady-state solutions for an n-pool system using a power-law approximation, J. Theoret. Biol. 25:370-379 (1969).

12 M. A. Savageau, Biochemical systems analysis III. Dynamic solutions using a power-law approximation, $J$. Theoret. Biol. 26:215-226 (1970).

13 M. A. Savageau, Parameter sensitivity as a criterion for evaluating and comparing the performance of biochemical systems, Nature 229:542-544 (1971).

14 M. A. Savageau. Concepts relating the behavior of biochemical systems to their underlying properties. Arch. Biochem. Biophy's. 145:612-621 (1971).

15 M. A. Savageau, The behavior of intact biochemical control systems, Current Top. Cell. Reg. 6:63-130 (1972).

16 M. A. Savageau, Biochemical systems analysis: A Study of Function and Design in Molecular Biology, Addison-Wesley, Reading, Mass., 1976.

17 M. A. Savageau, Growth of complex systems can be related to the properties of their underlying detcrminants, Proc. Nat. Acad. Sci. U.S.A. 76(11):5413-5417 (1979).

18 M. A. Savageau, Allometric morphogenesis of complex systems: Derivation of the basic equations from first principles, Proc. Nat. Acad. Sci. U.S. A. 76(12):6023-6025 (1979).

19 M. A. Savageau, Growth equations: A general equation and a survey of special cases, Math. Biosci. 48:267-278 (1980).

20 M. A. Savageau, A suprasystem of probability distributions, Biometrical J. 24:323-330 (1982).

21 M. A. Savageau and E. O. Voit, Power-law approach to modeling biological systems. I. Theory, J. Ferment. Technol. 60:221-228 (1982).

22 E. O. Voit and M. A. Savageau, Power-law approach to modeling biological systems II. Application to ethanol production, J. Ferment. Technol. 60:229-232 (1982).

23 E. O. Voit and M. A. Savageau, Power-law approach to modeling biological systems. III. Methods of analysis, J. Ferment. Technol. 60:233-241 (1982).

24 E. O. Voit and M. A. Savageau, Analytical solutions to a generalized growth equation, J. Math. Anal. Appl. 103:380-386 (1984).

25 E. O. Voit and M. A. Savageau, Accuracy of alternative strategies for the power-law modeling of biochemical systems, in preparation.

26 E. O. Voit and M. A. Savageau, in preparation.

27 V. Volterra, Leçons sur la Théorie Mathématique de la Lutte pour la Vie, GauthierVillars, Paris, 1931. 